\title{
miR-182 promotes cell growth and invasion by targeting forkhead box $F 2$ transcription factor in colorectal cancer
}

\author{
YU ZHANG $^{1 *}$, XINYING WANG ${ }^{2 *}$, ZHONGQIU WANG $^{2}$, HUI TANG $^{1}$, HONG FAN $^{1}$ and QIANG GUO $^{1}$ \\ ${ }^{1}$ Yunnan Provincial Institute of Digestive Medicine, Department of Gastroenterology, The First People's Hospital \\ of Yunnan Province, Kunming; ${ }^{2}$ Guangdong Provincial Key Laboratory of Gastroenterology, \\ Department of Gastroenterology, Nanfang Hospital, Southern Medical University, Guangzhou, P.R. China
}

Received September 26, 2014; Accepted January 30, 2015

DOI: $10.3892 /$ or.2015.3833

\begin{abstract}
Forkhead box F2 transcription factor (FoxF2) has been described to promote organ development, extracellular matrix (ECM) synthesis and epithelial-mesenchymal interaction. Although recent studies reported decreased FoxF2 expression in several types of cancers, indicating its potential role in carcinogenesis, the mechanistic role of FoxF2 is yet to be explored. MicroRNAs (miRNAs) are strongly implicated in carcinogenesis. The oncogenetic properties of miR-182 have been described in multiple cancers. In the present study, we aimed to investigate the role of miR-182 in colorectal cancer (CRC) and identify the regulation of FoxF2 by miR-182. Bioinformatic analyses on gene expression profiling datasets showed decreased FoxF2 expression in colorectal adenomas, primary tumors compared to normal colon epithelial and a negative association between FoxF2 and $\beta$-catenin expression. Restoration of FoxF2 in CRC cells suppressed $\beta$-catenin expression and simultaneously inhibited cell growth and invasion. Furthermore, we observed that miR-182 was aberrantly upregulated in CRC. Knockdown of miR-182 in CRC cells impeded cell growth and invasion. The direct binding of miR-182 to the $3^{\prime}$ untranslated region (3'UTR) of FoxF2 mRNA was confirmed using a luciferase reporter gene assay. Importantly, elevated FoxF2 expression was observed in miR-182-knockdown cells with a simultaneous reduction in $\beta$-catenin. In conclusion, the present study describes a potential mechanism underlying an miR-182/FoxF2 link contributing to CRC development. miR-182-induced downregulation of FoxF2 partly accounts for increased activity of $\beta$-catenin signaling. Inhibition of miR-182 represents a potential strategy against CRC.
\end{abstract}

Correspondence to: Professor Qiang Guo, Yunnan Provincial Institute of Digestive Medicine, Department of Gastroenterology, The First People's Hospital of Yunnan Province, Kunming, P.R. China E-mail: gqkj003@sina.com

${ }^{*}$ Contributed equally

Key words: microRNA, miR-182, colorectal cancer, FoxF2, cell invasion

\section{Introduction}

Forkhead transcription factors are characterized by a winged helix DNA-binding domain and are essential for embryogenesis (1). Some of the members have been identified to regulate tumorigenesis and progression, such as FoxO1 and FoxQ1 (2-4). Decreased forkhead box F2 transcription factor (FoxF2) expression was reported in prostate and breast cancer and was found to correlate with early-onset metastasis and poor prognosis $(5,6)$, which implies the potential role of FoxF2 in tumorigenesis and progression. Mechanistically, Ormestad et al found that FoxF2 regulates embryonic gut development by controlling paracrine crosstalk between the stroma and the epithelia (7). Inhibition of FoxF2 was found to increase the mesenchymal expression of Wnt5a, which subsequently activates the canonical Wnt signaling pathway to cause epithelial depolarization and tissue disintegration. Notably, a quite recent study demonstrated that mesenchymal FoxF2 restricted Lgr5-positive stem cells of the crypt niche and reduced adenoma formation in mouse intestines by inhibiting Wnt signaling (8). These results partly unveiled the mechanism that underlies the initiation of intestinal carcinoma by silencing of FoxF2 and indicate a tumor-suppressive role. Accordingly, it would be worthy to determine the upstream regulators and downstream effectors of the FoxF2 gene in tumor development.

MicroRNAs (miRNAs) are a cluster of small non-coding RNA molecules 22 nucleotides in length. They repress the expression of target genes by inducing mRNA degradation or by blocking translation. Emerging evidence suggests that miRNAs are involved in tumor development by functioning as oncogenes or tumor suppressors, depending on their target genes and downstream signaling pathways. miR-182 has been found to be aberrantly overexpressed and promotes tumorigenesis in breast, ovarian and prostate cancer (9-12). Moreover, miR-182 was also found to facilitate metastasis in melanoma and breast cancer through suppression of FOXO3 and MTSS1, respectively $(13,14)$. Importantly, Moskwa et al reported that miR-182 is implicated in DNA damage response (15). They observed that overexpression of miR-182 in sporadic breast cancer impeded homologous recombination-mediated DNA repair by targeting BRCA1, but rendered tumor cells hypersensitive to (ADP-ribose) polymerase 1 (PARP1) inhibitor 
treatment. This indicates the potential of miR-182 as a therapeutic target.

As shown in a meta-analysis, colorectal cancer (CRC) consistently exhibits aberrant overexpression of miR-182 (16), which suggests its potential oncogenic role in CRC. A recent study based on a comprehensive miRNA library screen showed that aberrantly overexpressed miR-182 promotes cell proliferation and survival in $\mathrm{CRC}$, and revealed a significant inverse correlation of FoxF2 to miR-182 by employing gene expression profiling data and target prediction tools (17). We thus hypothesized that aberrant expression of miR-182 in CRC promotes tumor development by suppressing FoxF2.

\section{Materials and methods}

Tissue samples and cell lines. A panel of 33 pairs of primary CRCs and their matched adjacent normal mucosa, and another independent panel of 49 specimens including 10 normal colon tissues and 39 CRCs classified in different stages, were obtained from patients who underwent surgical resections at the First People's Hospital of Yunnan Province, and were snap-frozen in liquid nitrogen, and then stored at $-80^{\circ} \mathrm{C}$ for further use. This project was approved by the Ethics Committee of the First People's Hospital of Yunnan Province.

Human CRC cell lines, including HT29, SW480, SW620 and HCT116, and human normal colon epithelial cell line FHC, were purchased from the American Type Culture Collection (ATCC). CRC cells were cultured routinely in RPMI-1640 medium (Invitrogen) supplemented with $10 \%$ fetal bovine serum (Gibco) and cultured in a $37^{\circ} \mathrm{C}$ humidified atmosphere of $5 \% \mathrm{CO}_{2}$. FHC cells were cultured following the instructions of the ATCC.

Bioinformatic analyses. All analyses of microarray datasets were performed using bioinformatic tool R2 (http://r2.amc.nl) following the instructions.

Lentivirus packaging and stable cell line establishment. The miR-182-knockdown or FoxF2-expressing lentivirus particles were packaged by co-transfecting the miR-182 inhibitor clone or the FoxF2-expressing clone with lentiviral packaging plasmids into HEK293FT cells, using Lenti-Pac ${ }^{\mathrm{TM}}$ HIV Expression Packaging Systems (both from GeneCopoeia) according to the manufacturer's instructions. For the lentivirus infection, cells were incubated with viral supernatant in the presence of $8 \mu \mathrm{g} / \mathrm{ml}$ Polybrene for $24 \mathrm{~h}$, followed by hygromycin or puromycin (Invitrogen) selection until drug-resistant colonies became visible.

RNA extraction and quantitative RT-PCR. Total RNA and miRNA were isolated using the RNeasy ${ }^{\circledR}$ Mini kit and miRNeasy ${ }^{\circledR}$ Mini kit (Qiagen) respectively. Mature miR-182 expression in cells was determined using the Hairpin-it ${ }^{\mathrm{TM}}$ miRNAs qPCR kit (GenePharma, China). RNU6B was used as an endogenous control. mRNA expression was determined using the SYBR-Green qPCR assay (Takara). Data were analyzed using the $2^{-\Delta \Delta \mathrm{Ct}}$ method.

Cell growth and invasion assays. Cell growth was detected using an MTT cell proliferation assay kit (ATCC) according to the manufacturer's instructions. Cell invasion ability was measured using Transwell inserts with $8-\mu \mathrm{m}$ pores (Corning). Briefly, the cell density was adjusted to $10^{6} / \mathrm{ml}$ in serum-free RPMI-1640 medium. The cell suspension (200 $\mu \mathrm{l})$ was added into each upper insert pre-coated with Matrigel matrix (BD), while $500 \mu$ l RPMI-1640 medium containing $10 \%$ fetal bovine serum (FBS) was added into a matched lower chamber. After a 48-h incubation, the non-invaded cells were removed from the upper surface of the Transwell membrane with a cotton swab, while the invaded cells on the lower membrane surface were fixed in methanol, stained with $0.1 \%$ crystal violet, photographed and counted. Six random fields at x100 magnification for each insert were observed.

Western blot analysis. Cultured cells were lysed in RIPA buffer with $1 \%$ phenylmethylsulphonyl fluoride (PMSF). Protein was loaded onto an SDS-PAGE Minigel and transferred onto a PVDF membrane. After being probed with 1:500 diluted anti-FoxF2 (Abcam) and 1:1,000 diluted anti- $\beta$-catenin (BD) at $4^{\circ} \mathrm{C}$ overnight, the blots were subsequently incubated with HRP-conjugated anti-IgG (Cell Signaling). Signals were visualized using ECL substrates (Pierce). $\alpha$-tubulin (Santa Cruz) was used as an endogenous protein for normalization.

Colony formation assay. Cells were trypsinized and plated on 6-well plates (300 cells for each well) and cultured for 10 days. The colonies were stained with $0.1 \%$ crystal violet solution containing $80 \%$ methanol for $5 \mathrm{~min}$. The number of colonies defined as $>50$ cells/colony were counted.

Luciferase reporter assay. A fragment of the wild-type 3' untranslated region (3'UTR) of FoxF2 containing the putative miR-182 binding site was amplified by PCR. The PCR product was subcloned into a psiCHECK-2 vector (Promega) immediately downstream to the luciferase gene sequence. A corresponding psiCHECK-2 construct containing 3'UTR of FoxF2 with a mutant seed sequence of miR-82 was also synthesized.

HEK293 cells were plated in 96-well clusters, and then co-transfected with $100 \mathrm{ng}$ constructs with or without miR-182 mimics. At $48 \mathrm{~h}$ post-transfection, luciferase activity was detected using a Dual-Luciferase Reporter Assay System (Promega) and normalized to Renilla activity.

Statistical analyses. Statistical analyses were performed using the SPSS 15.0.0 and GraphPad Prism software. Comparisons between the two groups were performed by the Student's t-test or the Mann-Whitney U test. Comparisons among multiple groups were performed by one-way ANOVA. A P-value of $<0.05$ was considered to indicate a statistically significant result.

\section{Results}

FoxF2 expression is downregulated in CRC and is negatively associated with $\beta$-catenin. FoxF 2 has been reported to reduce intestinal adenoma formation, by antagonizing the activity of the Wnt/ $\beta$-catenin pathway through upregulating the expression of SFRP1 which encodes an inhibitor of Wnt (8), yet knowledge concerning FoxF2 in human CRC 
A

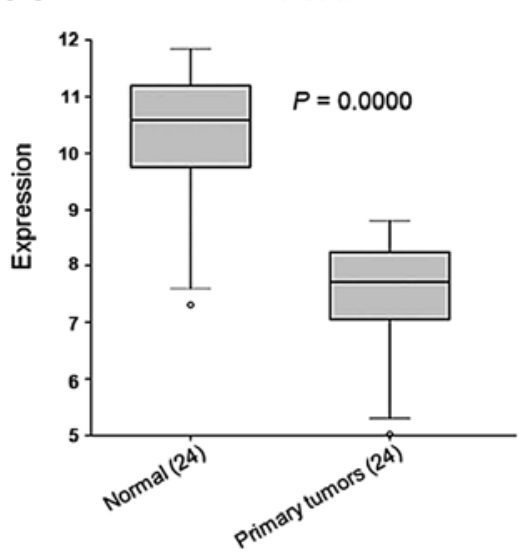

C

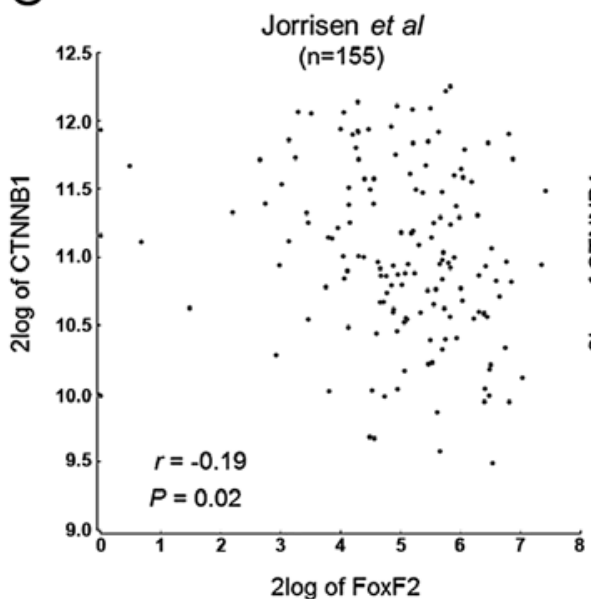

Sabates-Bellver et al
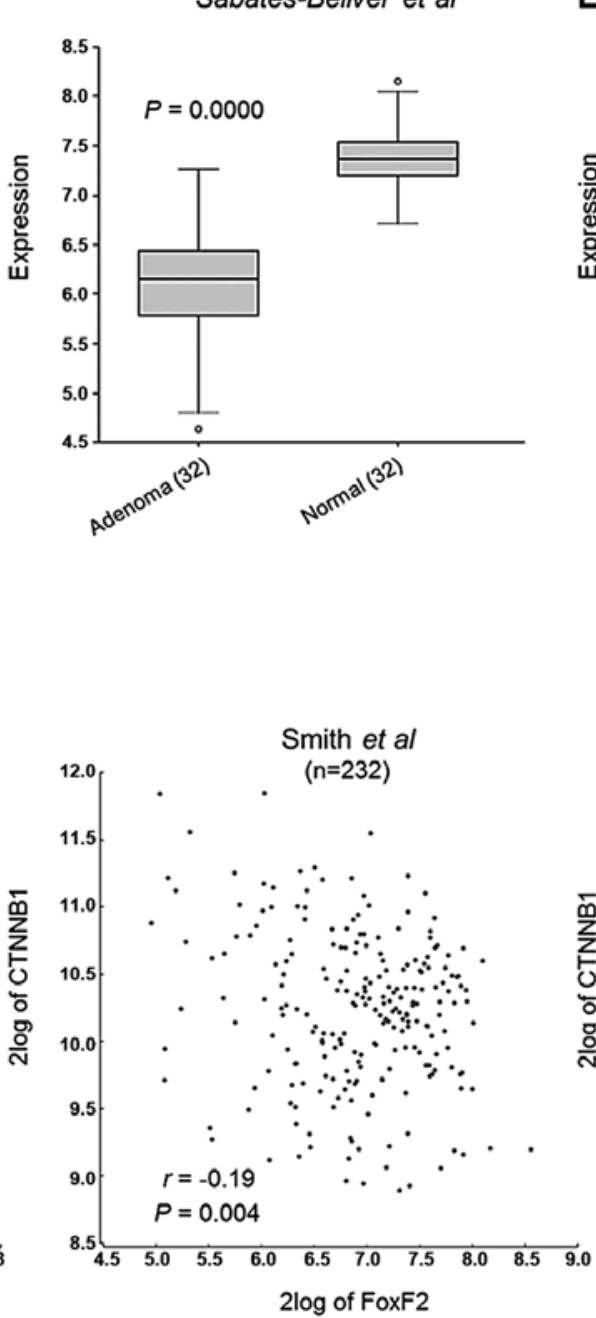

B
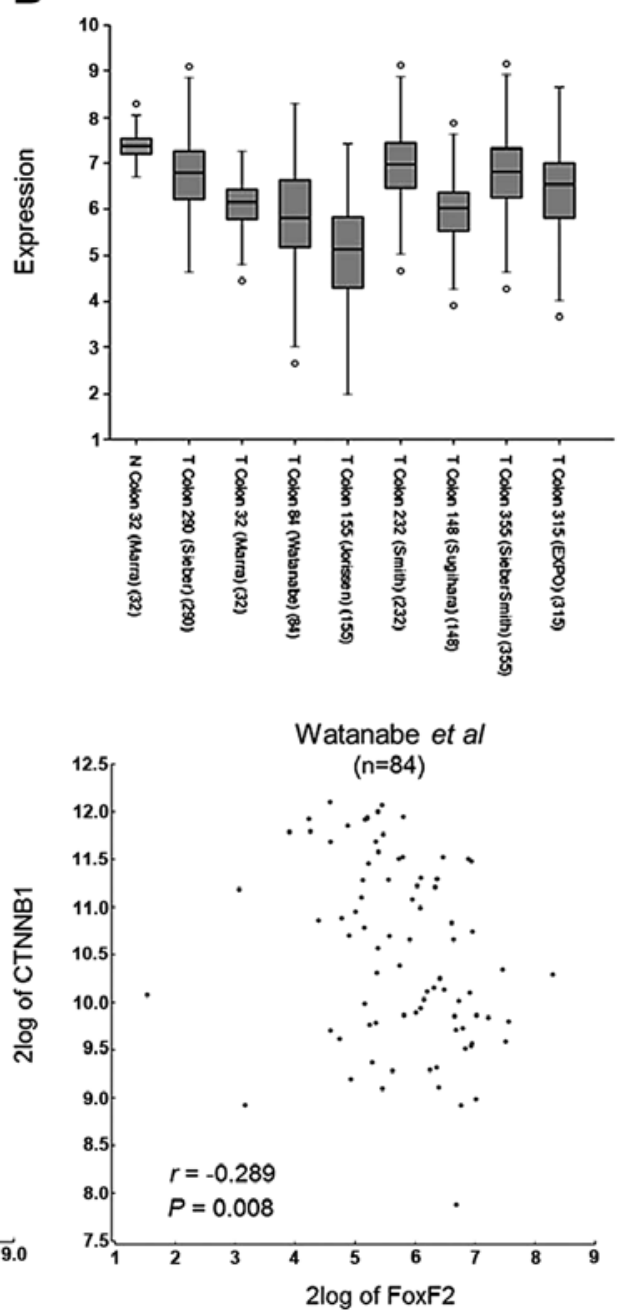

Figure 1. Downregulation of FoxF2 in CRC and its negative association with $\beta$-catenin. (A) Decreased mRNA level of FoxF2 in colorectal adenomas or primary CRCs, compared with paired normal colon epithelia, is plotted based on two gene expression profiling datasets of human CRC cohorts. (B) In an analysis of multiple human CRC datasets, FoxF2 is shown to be commonly decreased in all the tumor cohorts compared to a normal colon epithelia cohort. (C) FoxF2 expression in $\mathrm{CRC}$ was found to be negatively associated with $\beta$-catenin expression by analyzing 3 independent $\mathrm{CRC}$ cohorts. $\mathrm{CRC}$, colorectal cancer.

is sparse. Therefore, we first analyzed the gene expression profiles of different human CRC cohorts to observe the status of FoxF2 expression. As shown in Fig. 1A, FoxF2 was significantly downregulated in colorectal adenomas (18) and primary tumors (19) when compared with the paired normal colon tissues. In the case of multiple dataset analysis, FoxF2 expression was commonly lower in primary CRC, compared with the cohort of normal colon tissues [N Colon 32 (Mara) (18)] (Fig. 1B). Additionally, FoxF2 expression was observed to be negatively associated with oncogenic $\beta$-catenin expression in several datasets (20-23) (Fig. 1C).

Restoration of FoxF2 inhibits CRC cell growth and invasion by antagonizing $\beta$-catenin activity. We next attempted to investigate the role of FoxF2 in CRC progression and the potential mechanism. We screened the FoxF2 expression in different CRC cell lines, and observed a notable decrease in FoxF2 expression both at the mRNA and protein levels in CRC cells compared with the normal colon epithelial cell line FHC (Fig. 2A). Stable FoxF2-expressing CRC cells were generated by introducing FoxF2-expressing lentiviral particles into HCT116 and HT29 cells which express relatively lower FoxF2. In the gain-of-function assays, upregulation of FoxF2 reduced cell growth, colony formation (Fig. 2B and C) and impaired cell motility including migration (Fig. 2D) and invasion (Fig. 2E). By immunoblotting, in the FoxF2-expressing stable cells, $\beta$-catenin was downregulated upon the restoration of FoxF2 (Fig. 2F), demonstrating the antagonistic effect of FoxF2 on $\beta$-catenin activity in CRC.

miR-182 is aberrantly upregulated in CRC tissues and cell lines. To demonstrate the expression pattern of miR-182 in $\mathrm{CRC}$, we performed quantitative PCR to detect the miR-182 level in both clinical specimens and cell lines. In the cohort of 33 cases of paired CRC tissues and adjacent colon epithelia, miR-182 was significantly upregulated in 26 (78.8\%) tumors compared to that in the matched normal epithelia (Fig. 3A). In further analysis of miR-182 expression in 49 colon specimens (10 normal colon tissues and 39 CRCs diagnosed at different clinical stages), miR-182 in the advanced stage CRCs 

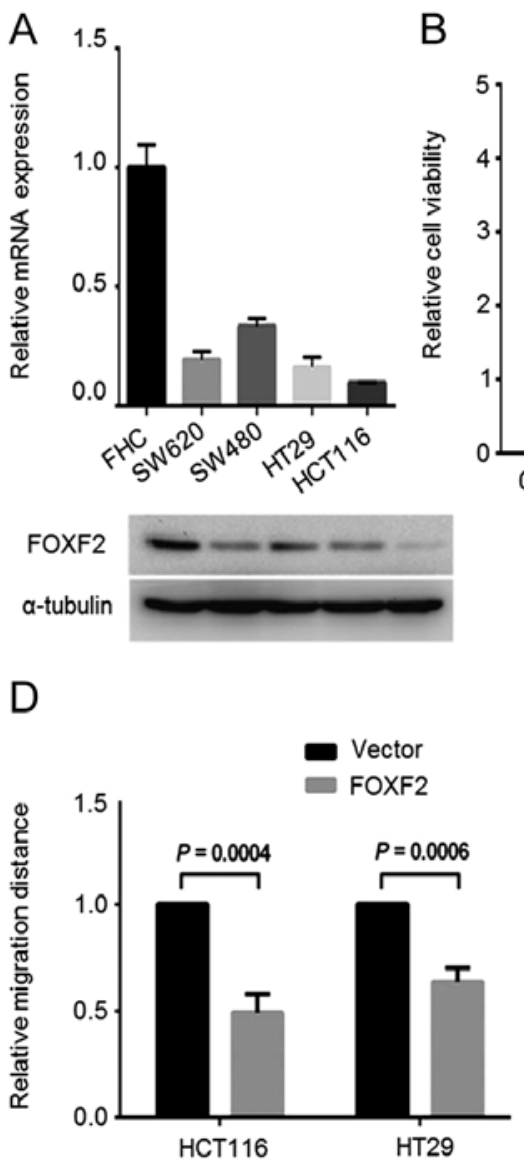

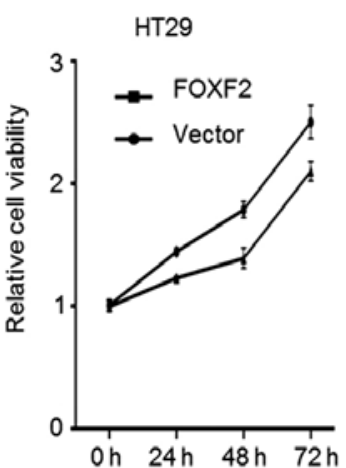

C
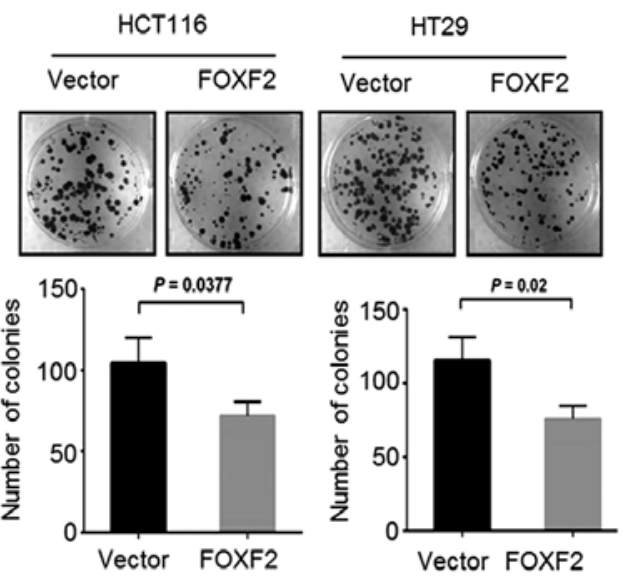

$E$

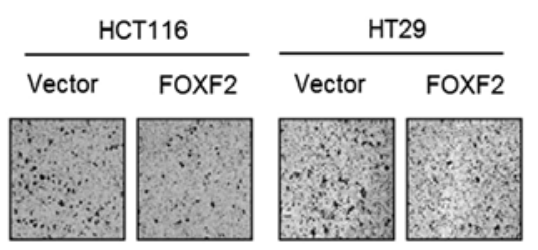

$\mathrm{F}$
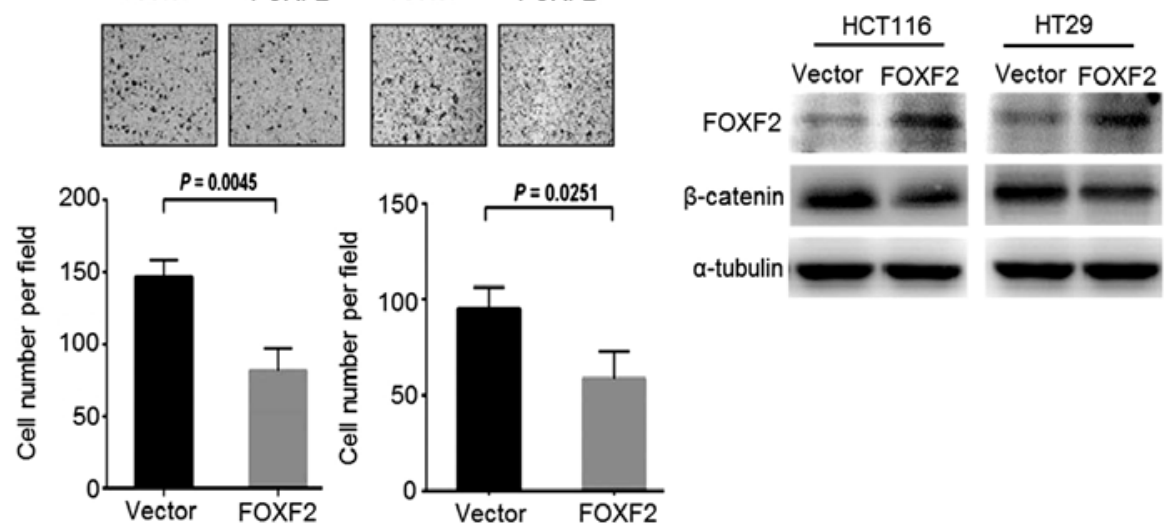

Figure 2. Tumor suppressive properties of FoxF2 in CRC cell lines. (A) Screening of FoxF2 expression at both the mRNA and protein levels in CRC cell lines. FoxF2 is significantly silenced in the CRC cell lines compared with the FHC cells (a normal colon epithelial cell line). Introduced FoxF2 restoration in HCT116 and HT29 cells simultaneously suppressed (B) cell growth, (C) colony formation, (D) cell migration and (E) invasion. (F) By western blotting, reduced $\beta$-catenin expression was observed in both FoxF2-expressing HCT116 and HT29 cell lines. CRC, colorectal cancer.

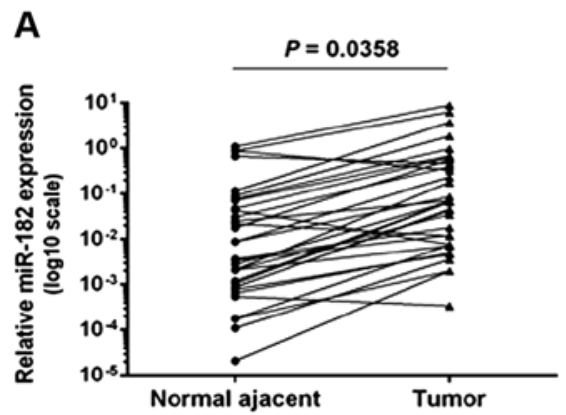

B

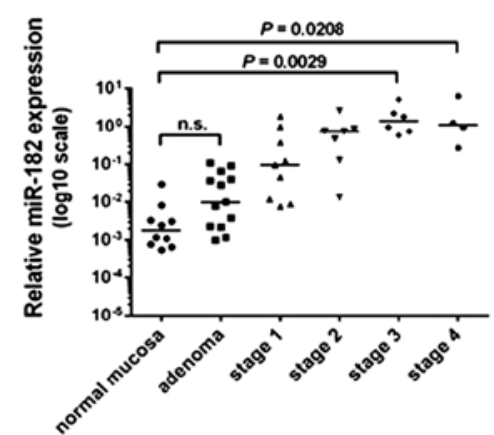

C

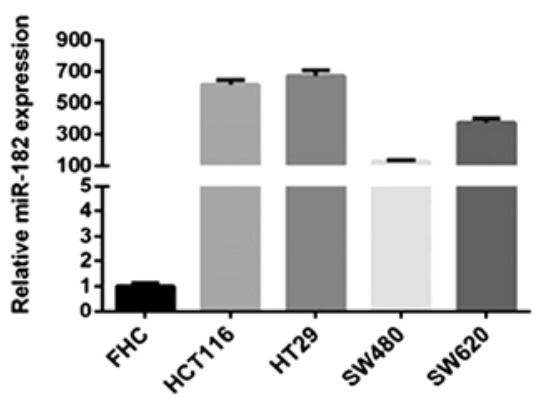

Figure 3. Aberrant upregulation of miR-182 in CRC tissues and cell lines. (A) Upregulated miR-182 expression is observed in CRCs compared to the adjacent normal colon tissues from a panel of $33 \mathrm{CRCs}$ (Wilcoxon's paired test). (B) In another panel of CRCs at different clinical stages, miR-182 was highly expressed in advanced stage CRCs (III and IV) in comparison with normal colon tissues and early stage tumors. In addition, no alteration of miR-182 expression was observed in colon adenomas. (C) miR-182 was commonly upregulated in all indicated CRC cell lines, while FHC cells expressed a low level of miR-182. CRC, colorectal cancer.

(III and IV) was significantly increased compared to the normal colon tissues and the early stage tumors, while no alteration of miR-182 level was observed in the adenomas (Fig. 3B).
We further examined the miR-182 expression level in the CRC cell lines: HCT116, HT29, SW480 and SW620, in comparison with that in the normal colonic epithelia cell line 
A

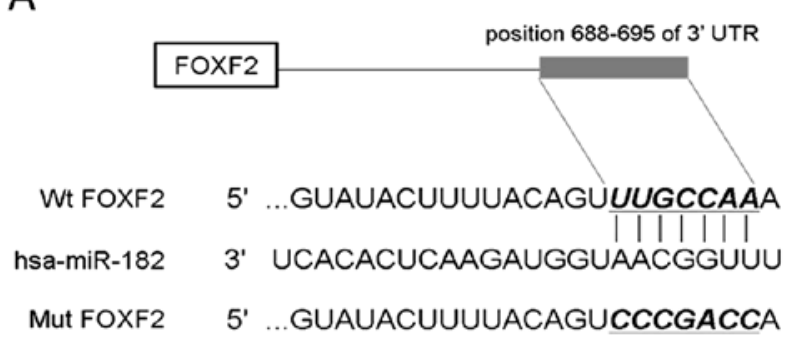

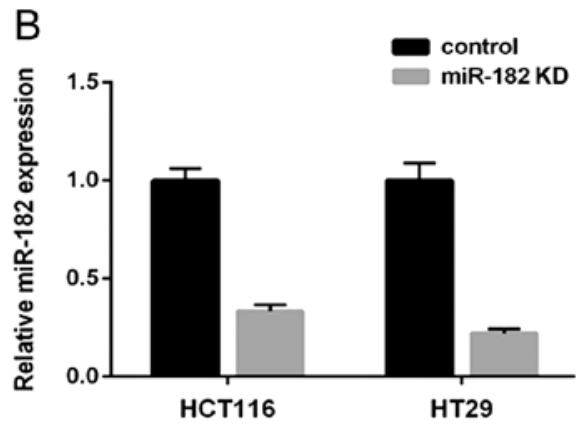

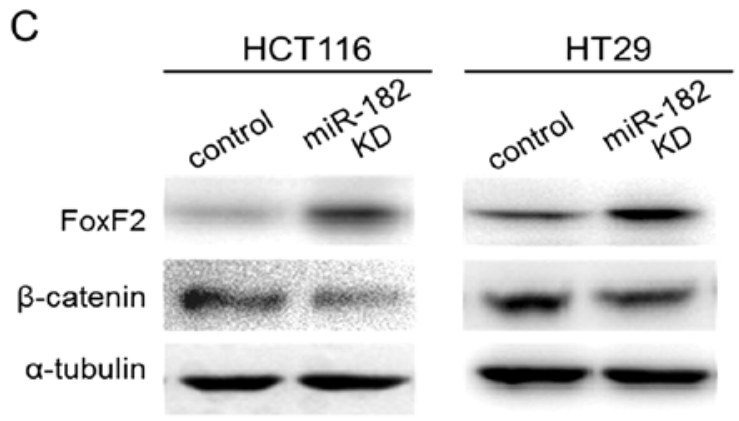

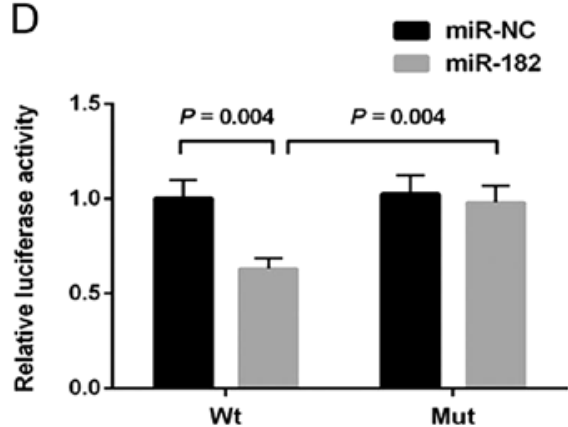

Figure 4. FoxF2 is a direct target of miR-182. (A) Predicted miR-182 binding site within FoxF2 3'UTR and its mutated version are as shown. (B) Downregulation of miR-182 is validated by qPCR in miR-182-knockdown HCT116 and HT29 cells. (C) Downregulation of miR-182 induces elevated expression of FoxF2. (D) Repression of luciferase activity by FoxF2 3'UTR is dependent on miR-182. Mutated 3'UTR of FoxF2 abrogates miR-182-mediated repression of luciferase activity. CRC, colorectal cancer; 3'UTR, 3' untranslated region.

FHC. As expected, miR-182 was consistently overexpressed in all of the 4 CRC cell lines (Fig. 3C). Since HCT116 and HT29 cells expressed relatively high levels of miR-182, they were selected for further functional assays.

FoxF2 is a direct target gene of miR-182 in CRC. In an integrative analysis, miR-182 was predicted to inversely correlate with FoxF2 that has a putative binding site of miR-182 (17). A recent study on prostate cancer further validated that miR-182 could target FoxF2 to promote cell invasion and proliferation (24). By utilizing bioinformatic algorithms (Targetscan/Pictar/miRecords), we noted that FoxF2 was a highly potential target gene of miR-182. The predicted binding site of miR-182 within 3'UTR of FoxF2 mRNA is shown in Fig. 4A. Since CRC cell lines exhibited a significant decrease in FoxF2 expression both at the mRNA and protein levels, we thus hypothesized a link between miR-182 and FoxF2. For further assays, we generated stable miR-182-knockdown CRC cells by infecting HCT116 and HT29 cells with lentiviral particles. The efficiency of knockdown of miR-182 expression was validated by qPCR (Fig. 4B). Immunoblotting showed that FoxF2 was notably upregulated in the miR-182-knockdown HCT116 and HT29 cells, while reduced $\beta$-catenin was also observed simultaneously (Fig. 4C). To further investigate whether the predicted binding site of miR-182 to 3'UTR of FoxF2 is responsible for the upregulation, we cloned the $3^{\prime} \mathrm{UTR}$ of FoxF2 into a luciferase reporter vector (wt-FoxF2); a corresponding mutant version (mut-FoxF2) was also constructed. We co-transfected wt-FoxF2 and miR-182 mimics or scramble control into the HEK293 cells. The luciferase activity of the miR-182-transfected cells was significantly reduced compared to the scramble control cells (Fig. 4D). Moreover, miR-182-mediated repression of luciferase activity was abolished by the mutant putative binding site (Fig. 4D).

Knockdown of miR-182 suppresses CRC cell growth and invasion in vitro. To explore whether miR-182 contributes to oncogenic phenotypes of CRC, we performed loss-of-function assays on miR-182-knockdown CRC cells. By an MTT assay, we observed that downregulation of miR-182 significantly reduced the cell growth of CRC cells compared to the scramble control cells (Fig. 5A). Similarly, we also observed a decrease in colony formation activity in the miR-182-knockdown cells (Fig. 5B). These findings were similar to those presented for the stable FoxF2-expressing CRC cells. Furthermore, we performed wound healing and Transwell invasion assays to investigate the involvement of miR-182 in cell motility of CRC. As shown in Fig. 5C and D, downregulation of miR-182 significantly reduced the cell migration and cell invasive capacity of CRC cells to migrate through Matrigel. These findings indicate that aberrant upregulation of miR-182 is prooncogenic and may confer malignant potential to CRC cells.

\section{Discussion}

Growing evidence has linked the expression of miR-182 to multiple types of tumors including prostate cancer, melanoma, breast cancer and glioma (12-14,25), although studies have reported different, even contradictory, roles of miR-182 in tumorigenesis in different tumor models $(11,14,26)$. In CRC, miR-182 was shown to be aberrantly upregulated as well as in precursor lesions (27), and its upregulation was significantly 

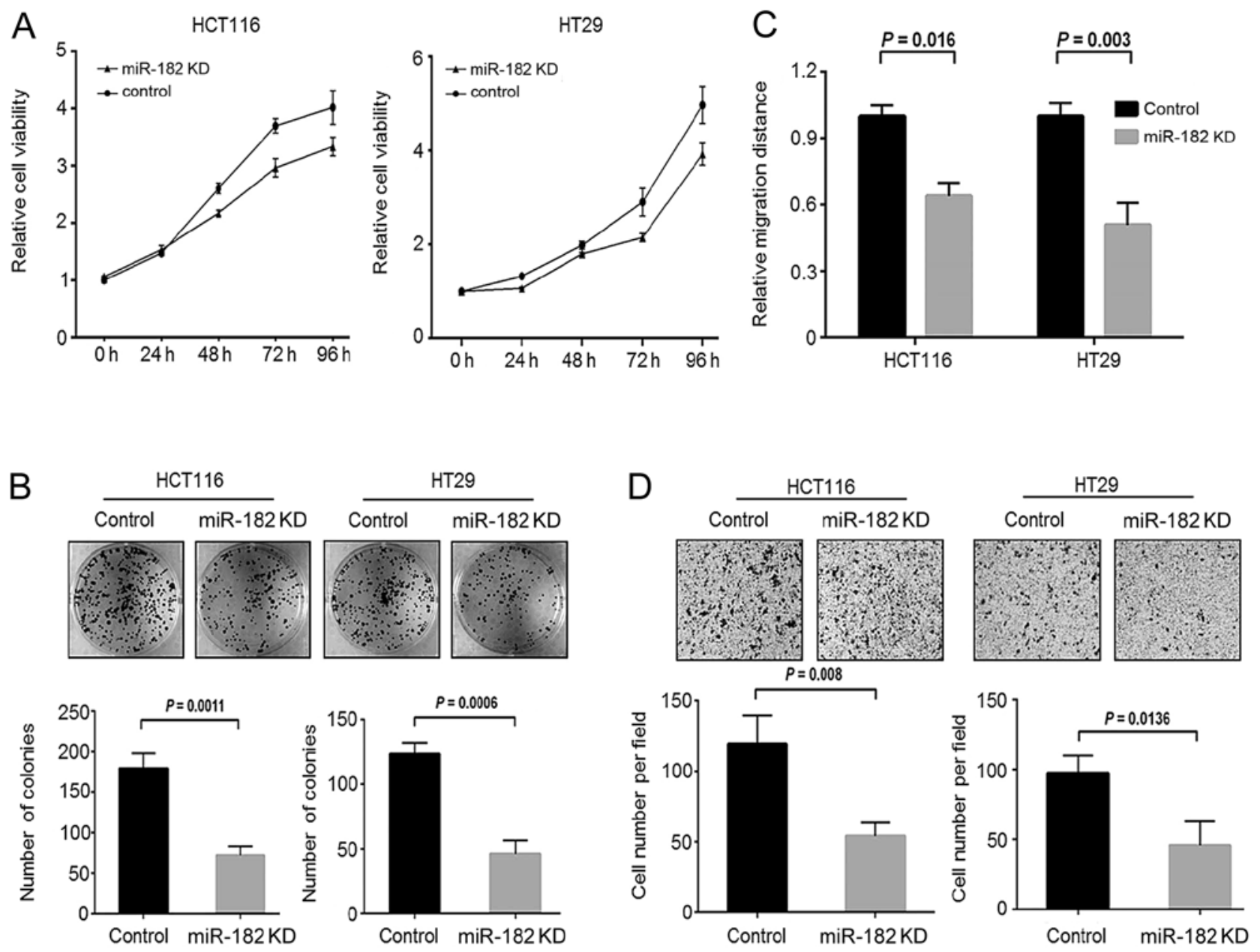

Figure 5. Functional effects of miR-182 downregulation on CRC cells. Downregulation of miR-182 inhibits (A) cell growth, (B) colony formation activity, (C) cell migration and (D) cell invasion ability of the HCT116 and HT29 cells. CRC, colorectal cancer.

associated with lymph node metastasis, TNM stage and poor prognosis (28). Diep et al reported that the miR-182 gene was amplified in $26 \%$ of primary CRC tumors and $30 \%$ of liver metastases (29). It was further demonstrated that miR-182 reduced the apoptosis of CRC cells by performing RNAi screening and functional validation (17), which functionally suggests the oncogenic role of miR-182 in CRC. In the present study, we observed the increased expression of miR-182 in adenomas and primary CRCs compared to normal colon mucosa. Moreover, increased miR-182 was correlated with advanced tumor stage, which is similar to the previous study. Simultaneous suppression of in vitro cell growth, colony formation, migration, invasion of CRC cells is shown upon inhibition of miR-182. Collectively with the previous report, our results highlight the functional characteristics of miR-182 as an oncomiR in CRC.

Despite that miR-182 has been validated to target several tumor suppressors including PTEN, MTSS1, Slug and CYLD in different types of cancers, we sought to reveal the mechanistic link of miR-182 to target genes contributing to CRC development. We observed that FoxF2, a member of the forkhead box (FOX) family of transcription factors, is highly predicted as a miR-182 target using several bioinformatic algorithms. In previous studies, FoxF2 was described to promote organ development, extracellular matrix (ECM) synthesis and epithelial-mesenchymal interaction $(7,30,31)$. It can be activated by hedgehog signaling, and subsequently limits Bmp and Wnt signaling (7). FoxF2 in normal prostate stroma could facilitate stomal homeostasis through upregulation of CXCL12 and regulation of ECM remodeling via balancing MMPs and TIMPs (5). A recent study showed that a low level of FoxF2 mRNA is associated with early-onset metastasis and poor prognosis of patients with histological grade II and triple-negative breast cancer (6), indicating its clinical value as a prognostic marker. We demonstrated that FoxF2 is downregulated in primary CRC tissues and CRC cell lines. Restoration of FoxF2 in CRC cells significantly suppressed cell growth, colony formation and cell motility, indicating the tumor-suppressive role of FoxF2. Since mesenchymal FoxF2 restricted Lgr5-positive stem cells of the crypt niche and reduced adenoma formation in mouse intestines by inhibiting Wnt/ $\beta$-catenin signaling (8), we hypothesized that loss of FoxF2 may result in increased $\beta$-catenin expression and activity. Indeed, $\beta$-catenin expression was experimentally detected to decrease in the FoxF2-expressing CRC cells as well as in the miR-182-knockdown cells. Given that miR-182 binds to the 3'UTR of FoxF2 mRNA thus leading to a decrease in FoxF2 expression, we demonstrated that aberrantly elevated miR-182 is responsible, at least partly, for the FoxF2 silencing and increased $\beta$-catenin activity in CRC. 
More intriguingly, recent studies have shown that miR-182 stimulates nuclear $\beta$-catenin translocation through repressing SMAD4 in bladder cancer (32), while miR-182 is transactivated by $\beta$-catenin in breast cancer (10), indicating the important role of the $\beta$-catenin/miR-182 feedback loop in tumor progression. Our results provide evidence for FoxF2 as a mediator involved in this oncogenic loop. In addition, miR-182 is shown to repress RECK, an antagonist of matrix metalloproteinases (MMPs), while FoxF2 upregulated the expression of the tissue inhibitor of metalloproteinase 3 (TIMP3), another strong antagonist of MMPs (5). This suggests that the miR-182/FoxF2 link may play a role in regulating ECM remodeling to facilitate tumor cell invasion and metastasis.

In conclusion, the present study identifies the regulatory link between miR-182 and FoxF2, and describes a potential mechanism underlying FoxF2 dysregulation and its contribution to CRC progression. Additionally, miR-182-induced downregulation of FoxF2 may partly account for the increased activity of $\beta$-catenin signaling. Inhibition of miR-182 represents a potential strategy against CRC.

\section{Acknowledgements}

The present study was supported by grants from the National Natural Science Foundation of China (81260323), and the Joint Foundation of Kunming Medical University and Yunnan Provincial Science and Technology Department (2012FB095).

\section{References}

1. Kaufmann E and Knöchel W: Five years on the wings of fork head. Mech Dev 57: 3-20, 1996.

2. Dong XY, Chen C, Sun X, Guo P, Vessella RL, Wang RX, Chung LW, Zhou W and Dong JT: FOXO1A is a candidate for the 13 q14 tumor suppressor gene inhibiting androgen receptor signaling in prostate cancer. Cancer Res 66: 6998-7006, 2006.

3. Zhang H, Meng F, Liu G, Zhang B, Zhu J, Wu F, Ethier SP, Miller F and Wu G: Forkhead transcription factor Foxq1 promotes epithelial-mesenchymal transition and breast cancer metastasis. Cancer Res 71: 1292-1301, 2011.

4. Qiao Y, Jiang X, Lee ST, Karuturi RK, Hooi SC and Yu Q: FOXQ1 regulates epithelial-mesenchymal transition in human cancers. Cancer Res 71: 3076-3086, 2011.

5. Van der Heul-Nieuwenhuijsen L, Dits N, Van Ijcken W, de Lange D and Jenster G: The FOXF2 pathway in the human prostate stroma. Prostate 69: 1538-1547, 2009.

6. Kong PZ, Yang F, Li L, Li XQ and Feng YM: Decreased FOXF2 mRNA expression indicates early-onset metastasis and poor prognosis for breast cancer patients with histological grade II tumor. PLoS One 8: e61591, 2013.

7. Ormestad M, Astorga J, Landgren H, Wang T, Johansson BR, Miura N and Carlsson P: Foxf1 and Foxf 2 control murine gut development by limiting mesenchymal Wnt signaling and promoting extracellular matrix production. Development 133: 833-843, 2006

8. Nik AM, Reyahi A, Pontén F and Carlsson P: Foxf2 in intestinal fibroblasts reduces numbers of $\mathrm{Lgr}^{+}$stem cells and adenoma formation by inhibiting Wnt signaling. Gastroenterology 144: 1001-1011, 2013

9. Guttilla IK and White BA: Coordinate regulation of FOXO1 by miR-27a, miR-96, and miR-182 in breast cancer cells. J Biol Chem 284: 23204-23216, 2009.

10. Chiang $\mathrm{CH}$, Hou MF and Hung WC: Up-regulation of miR-182 by $\beta$-catenin in breast cancer increases tumorigenicity and invasiveness by targeting the matrix metalloproteinase inhibitor RECK. Biochim Biophys Acta 1830: 3067-3076, 2013.

11. Liu Z, Liu J, Segura MF, Shao C, Lee P, Gong Y, Hernando E and Wei JJ: MiR-182 overexpression in tumourigenesis of high-grade serous ovarian carcinoma. J Pathol 228: 204-215, 2012.
12. Tsuchiyama $\mathrm{K}$, Ito $\mathrm{H}$, Taga $\mathrm{M}$, Naganuma $\mathrm{S}$, Oshinoya $\mathrm{Y}$, Nagano K, Yokoyama O and Itoh H: Expression of microRNAs associated with Gleason grading system in prostate cancer: miR-182-5p is a useful marker for high grade prostate cancer. Prostate 73: 827-834, 2013.

13. Segura MF, Hanniford D, Menendez S, et al: Aberrant miR-182 expression promotes melanoma metastasis by repressing FOXO3 and microphthalmia-associated transcription factor. Proc Natl Acad Sci USA 106: 1814-1819, 2009.

14. Lei R, Tang J, Zhuang X, Deng R, Li G, Yu J, Liang Y, Xiao J, Wang HY, Yang Q and Hu G: Suppression of MIM by microRNA-182 activates RhoA and promotes breast cancer metastasis. Oncogene 33: 1287-1296, 2014.

15. Moskwa P, Buffa FM, Pan Y, et al: miR-182-mediated downregulation of BRCA1 impacts DNA repair and sensitivity to PARP inhibitors. Mol Cell 41: 210-220, 2011.

16. Zhang QH, Sun HM, Zheng RZ, Li YC, Zhang Q, Cheng P, Tang ZH and Huang F: Meta-analysis of microRNA-183 family expression in human cancer studies comparing cancer tissues with noncancerous tissues. Gene 527: 26-32, 2013.

17. Cekaite L, Rantala JK, Bruun J, et al: MiR-9, -31, and -182 deregulation promote proliferation and tumor cell survival in colon cancer. Neoplasia 14: 868-879, 2012.

18. Sabates-Bellver J, Van der Flier LG, de Palo M, et al: Transcriptome profile of human colorectal adenomas. Mol Cancer Res 5: 1263-1275, 2007.

19. Jiang X, Tan J, Li J, et al: DACT3 is an epigenetic regulator of $\mathrm{Wnt} / \beta$-catenin signaling in colorectal cancer and is a therapeutic target of histone modifications. Cancer Cell 13: 529-541, 2008.

20. Jorissen RN, Lipton L, Gibbs P, et al: DNA copy-number alterations underlie gene expression differences between microsatellite stable and unstable colorectal cancers. Clin Cancer Res 14: 8061-8069, 2008

21. Smith JJ, Deane NG, Wu F, et al: Experimentally derived metastasis gene expression profile predicts recurrence and death in patients with colon cancer. Gastroenterology 138: 958-968, 2010.

22. Watanabe T, Kobunai T, Toda E, et al: Distal colorectal cancers with microsatellite instability (MSI) display distinct gene expression profiles that are different from proximal MSI cancers. Cancer Res 66: 9804-9808, 2006.

23. Tsukamoto S, Ishikawa T, Iida S, Ishiguro M, Mogushi K, Mizushima H, Uetake H, Tanaka H, Sugihara K: Clinical significance of osteoprotegerin expression in human colorectal cancer. Clin Cancer Res 17: 2444-2450, 2011

24. Hirata H, Ueno K, Shahryari V, Deng G, Tanaka Y, Tabatabai ZL, Hinoda Y and Dahiya R: MicroRNA-182-5p promotes cell invasion and proliferation by down regulating FOXF2, RECK and MTSS1 genes in human prostate cancer. PLoS One 8: e55502, 2013.

25. Jiang L, Mao P, Song L, Wu J, Huang J, Lin C, Yuan J, Qu L, Cheng SY and Li J: miR-182 as a prognostic marker for glioma progression and patient survival. Am J Pathol 177: 29-38, 2010.

26. Sun Y, Fang R, Li C, Li L, Li F, Ye X and Chen H: Hsa-mir-182 suppresses lung tumorigenesis through down regulation of RGS17 expression in vitro. Biochem Biophys Res Commun 396: 501-507, 2010.

27. Sarver AL, French AJ, Borralho PM, et al: Human colon cancer profiles show differential microRNA expression depending on mismatch repair status and are characteristic of undifferentiated proliferative states. BMC Cancer 9: 401, 2009.

28. Liu H, Du L, Wen Z, et al: Up-regulation of miR-182 expression in colorectal cancer tissues and its prognostic value. Int J Colorectal Dis 28: 697-703, 2013

29. Diep CB, Kleivi K, Ribeiro FR, Teixeira MR, Lindgjaerde OC and Lothe RA: The order of genetic events associated with colorectal cancer progression inferred from meta-analysis of copy number changes. Genes Chromosomes Cancer 45: 31-41, 2006.

30. Wang T, Tamakoshi T, Uezato T, Shu F, Kanzaki-Kato N, Fu Y, Koseki H, Yoshida N, Sugiyama T and Miura N: Forkhead transcription factor Foxf2 (LUN)-deficient mice exhibit abnormal development of secondary palate. Dev Biol 259: 83-94, 2003.

31. Aitola M, Carlsson P, Mahlapuu M, Enerback S and PeltoHuikko M: Forkhead transcription factor FoxF2 is expressed in mesodermal tissues involved in epithelio-mesenchymal interactions. Dev Dyn 218: 136-149, 2000.

32. Hirata H, Ueno K, Shahryari V, Tanaka Y, Tabatabai ZL, Hinoda Y and Dahiya R: Oncogenic miRNA-182-5p targets Smad4 and RECK in human bladder cancer. PLoS One 7: e51056, 2012 